\title{
Common features of gamma-radiation increase at different stations from Arctic to mid-latitudes
}

\author{
Yury Balabin ${ }^{1}$, Boris Gvozdevsky, Alexey Germanenko, Evgeny Mikhalko, Evgeny Maurchev, Leonid Shchur \\ Polar Gophysical Institute, 184209 Apatity, 26A Academgorodok, Russia.
}

\begin{abstract}
Background gamma-radiation (20-400 KeV) monitoring in the near-surface layer of the atmosphere has been carried out by Polar Geophysical Institute (PGI) for many years. This radiation originates in the atmosphere by cosmic rays. In propagation through the atmosphere, the flux of soft radiation experiences variations which are induced by various processes in the atmosphere. A unique and extensive database has been collected on the level of the soft gamma-radiation occurring in 2009-2017. Measurements are made with the help of similar detectors developed at PGI. All the stations observe the event which was for the first time discovered by the authors: gamma-background increase at precipitation. These increases are not related to any radionuclides in precipitation. Increases are observed only in the electromagnetic component originating in the atmosphere from cosmic rays. The complex analysis of the data collected has shown that the characteristics of the events (such as amplitude, duration, the count-rate, a total energy) have clear differences through the seasons at each station. The analysis of the events shows that variations of the background gamma-radiation can be used to study some atmospheric processes in the lower atmosphere.
\end{abstract}

\section{Introduction}

At present, monitoring of soft gamma-radiation $(20-400 \mathrm{KeV})$ coming from the atmosphere, is conducted, besides Apatity and Barentsburg, in Tiksi, Yakutsk, Mondy (the Sayan mountains), Rostov-on-Don. The monitoring is conducted with scintillation detectors $\mathrm{NaI}(\mathrm{Tl})$, developed in PGI. The detectors are shielded by lead bricks $5 \mathrm{~cm}$ thickness to reduce radiation from ground and environment [1]. Just from the beginning of observations, it was revealed that during precipitation, the gamma radiation flux increases by tens of percents. It happens all the year round. The relationship between precipitation and increase has been determined by special measurements made in Apatity. At this station, a precipitation gauge developed at the Laboratory of Arctic, PGI, [2], is included into the gamma-background monitoring system. The precipitation gauge is not gauged for making absolute measurements (the amount of precipitation, $\mathrm{mm} / \mathrm{hr}$ ), showing relative intensity of precipitation each minute. The virtue of this precipitation gauge is in that its data are produced each minute and are convenient for comparison with the data of detectors. Owing to the presence of the precipitation gauge the direct dependence between precipitation and increasing has been established. Also, it has been shown that the maximum of precipitation is at the front of increasing, and the maxima of increasing comes 30-40 minutes after the maximum of precipitation [3]. It has been also found out that increasing occurs only in an electromagnetic component, and the flux of charged particles (electrons, positrons, muons) does not increase [4-6]. With other variations of gamma-radiation (for example, a daily variation of 2-3\%) being observed, the following decision has been made: the changes in the gamma-background less than $5 \%$ are not considered as the event of increase.

${ }^{1}$ Corresponding author: balabin@pgia.ru 


\section{Statistical analysis of events}

Recorded for eight years are more than one and a half thousand increase events in Apatity, and more than four hundred events in Barentsburg. Other stations carry out observations for more than 2 years. It is enough for the statistical analysis of events to be carried out with the good accuracy. The data obtained during this period were processed, with all the events above the threshold of $5 \%$ being selected. The following parameters were determined: the amplitude of increasing, duration of the event, duration of the event front, the total energy which has been released during increasing, and some others. Then the distribution of the amount of events was built, depending on the value of the specified parameters. Figure 1 shows, as an example, typical events of gamma-radiation increasing at two stations.

Figure 2 shows the distribution of the amount of events, depending on the event amplitude. The background fluxes of gamma-radiation at the stations differ, being equal to 700-2800 pulses per minute. Therefore, the increases are measured and compared in absolute values. It is possible because detectors are of the same type and have equal spectral response. All stations are characterized by natural average frequency of the events occurrence. Therefore, the distribution is normalized to the amount of events at the station. The position of the maximum of the amplitude distribution at three stations (Yakutsk, Apatity, Tiksi) is on the 4-th bin (80-100 pulses/min). At the most northern station Barentsburg, the maximum is displaced to smaller values, in Rostov, it is replaced to greater values. A high-mountain station Hulugai in the Sayan mountains is characterized by the distribution with a wide plateau.
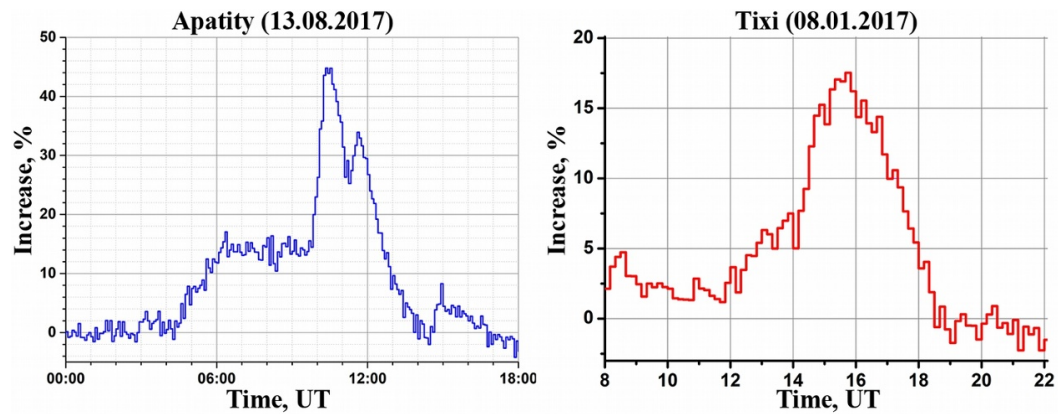

Fig.1. The profiles of the gamma-radiation increase events at Apatity station (left) and at Tiksi station (right). It is used the channel $1>20 \mathrm{KeV}$. The flux recorded the day before when the weather was good, is taken as a reference one.
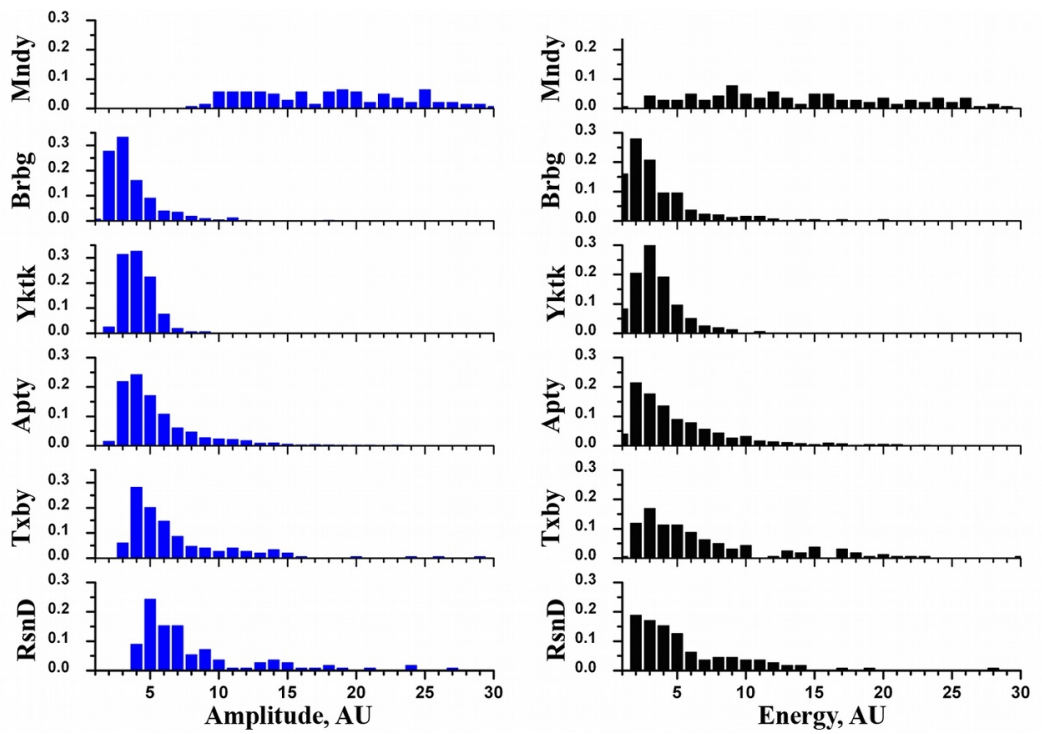

Fig.2. The events distribution by the amplitude (left) and by the total energy released with increasing (right). The stations: Mndy (Hulugai peak, $3000 \mathrm{~m}$ ), Brbg (Barentsburg), Yktk (Yakutsk), Apty (Apatity), Txby (Tiksi), RsnD (Rostov-on-Don). One bin in the $\mathrm{OX}$ in the left-hand graph corresponds to 20 pulses/min, a bin in the right graph corresponds to $2.5 \cdot 10^{4}$ quantum, with an average energy of $100 \mathrm{keV}$ of a quantum. 
The total amount of energy released with increasing, depends on both the amplitude of increasing and the event duration. Approximately, 50-60\% of events recorded at flat land stations, release no more than $10^{5}$ gamma-ray quantum, and only single extreme events release more than $2.5 \cdot 10^{5}$. The high-mountain station Hulugai drastically differs from the others: its distribution is very wide, with the events of $5 \cdot 10^{5}$ are not a scarcity for this station. Unlike amplitude distribution, in energy distributions, the shape, and especially the tail of distribution, is individual. The average quantum energy of $100 \mathrm{keV}$ is determined from the effective range of detectors (20-400 keV) and the average energy spectrum of gamma radiation in the near-surface layer of the atmosphere, measured with the same detector.

\section{Discussion}

The expansion of the network is used to observe the background gamma-radiation generated in the atmosphere from cosmic rays, allows making the conclusion that the phenomenon discovered at PGI, connected with the gamma-radiation flux increasing while precipitation, is universal rather than a singularity of the polar regions. Measurements made by typical detectors, as well as change from relative measurements (variations in \% against the background level at the given station) for absolute (pulse/minute) allowed establishing the fact that at all the flat land stations the increasing events parameters (amplitude, duration, the time of increase front etc.) are rather close; differ in much smaller extent if compared with the values of the background flux in good weather at these stations. Also based on these measurements, it is possible to make an assured and final conclusion that radon emanation from soil, under the phenomenon observed, are insignificant. At Tiksi station, the detector is placed on the roof of the laboratory building outdoor, at Hulugai station, the detector is placed in a small wooden structure on the crest of the mountain at the altitude of $3000 \mathrm{~m}$ where there are no windless days.

Thus, the gamma-radiation increase is the result of the processes occurring in the atmosphere and related to meteorological processes. Direct experiments have shown that these increases occur only in an electromagnetic component, with the flux of charged particles (electrons, positrons, muons) does not change in precipitation. As the basic one, the authors consider the hypothesis of accumulation of additional energy by charged particles in electric fields of the rain clouds with its subsequent radiation in Bremsstrahlung processes. In the rain (stratocumulus) clouds, there are electric fields of $\sim 10 \mathrm{kV} / \mathrm{m}$ [7]. The estimations made show that with typical thickness of a cloud of $1 \mathrm{~km}$ [8], the energy which can be accumulated by a charged particle, exceeds several times that coming to the detector. It means that in terms of energy, it is possible. However, other physical processes related to the particle transmission through a dense medium such as the lower atmosphere, strongly limit possible mechanisms generating electromagnetic radiation in the atmosphere. The most promising and non-contradicting mechanism is the "additional acceleration" effect: a highly energetic (tens of $\mathrm{MeV}$ ) light charged particle accumulates additional energy when passing through a rain cloud, losing it then in Bremsstrahlung processes.

\section{Conclusions}

The analysis of the gamma-radiation increase events with precipitation, which are recorded by a wide network of stations, is presented. The amount of events at each recently created station makes about one and a half hundreds and those at Apatity and Barentsburg stations, the amount is equal to1525 and 429, respectively.

The increase measurements in absolute values (pulses/minutes of the detectors counting) produce a more realistic idea. The temporal and energetic characteristics of these events in absolute measurements turned out to be rather close for all flat land stations located from $78^{\circ}$ to $47^{\circ}$ latitudes. The difference is available only at a high-mountain station Hulugai (the Sayan mountains).

The analytical results and the events distribution on the parameter value allow the conclusion that the phenomenon observed has a global character, takes place in the atmosphere everywhere, pointing out that the reasons of increase are physical and meteorological processes in the atmosphere, first of all, the electrical processes.

\section{References}

1. Yu. V. Balabin, A.V. Germanenko, B.B. Gvozdevsky, E.V. Vashenyuk, Bulletin of the Russian Academy of Sciences. Physics, 77, 575 (2013)

2. V.A.Shishaev, M.I.Beloglazov, Instrumentation and experimental techniques, 2, 156 (2011)

3. Yu. V. Balabin, A.V. Germanenko, 33rd ICRC proceeding, 2013 
4. A.V. Germanenko, Yu. V. Balabin, E.V. Vashenyuk, B.B. Gvozdevsky, L.I. Schur, Astrophys. Space Sci. Trans., 7, 471 (2011)

5. J.V.Balabin, A.V.Germanenko, B.B.Gvozdevsky, E.V.Vashenjuk, Geomagnetism and aeronomy, 54, 376 (2014)

6. A.V. Germanenko, Yu. V. Balabin, B.B. Gvozdevsky, L.I. Schur, Solar-Terrestrial Physics, 2, 78 (2016)

7. W.D. Rust, R.J. Trapp, Geophys. Res. Lett., 29, 1959 (2002)

8. L.T. Matveev, Fundamentals of General Meteorology: Physics of the Atmosphere (Israel Program for Scientific Translations, Jerusalem, 1968) 